\title{
Reflexiones sobre la importancia y diagnóstico del área estructural en programas de ingeniería civil en Colombia
}

\author{
Daniel Ricardo Salinas-Guayacundo
}

Facultad de Ingeniería Civil, Escuela Colombiana de Ingeniería Julio Garavito, Bogotá, Colombia. daniel.salinas@escuelaing.edu.co

\begin{abstract}
Resumen - En este artículo, la importancia del área estructural dentro de un programa académico de Ingeniería Civil es estudiada. Las características especiales de los programas en Ingeniería Civil en Colombia con énfasis en estructuras son tomadas como caso de estudio. Colombia es un país que está expuesto a una elevada amenaza sísmica además de poseer gran cantidad de edificaciones susceptibles a colapsar ante un evento sísmico. En los últimos 35 años, sismos moderados han destruido varias ciudades, han producido cientos de pérdidas humanas y materiales porque un número importante de sus edificaciones fueron diseñadas y construidas con poca o ninguna consideración de los efectos sísmicos. En este documento, tres programas en Ingeniería Civil (área estructural) fueron seleccionados y estudiados aleatoriamente estudiando dos aspectos (1) Docentes (formación y producción académica y contenidos programáticos), y (2) infraestructura (laboratorios y material de apoyo). Producto de lo anterior se encontró que el ítem de los profesores contribuye más en la calidad de la Universidad. Por lo tanto, aspectos relacionados con la formación de estudiantes colombianos en programas doctorales nacionales e internacionales son estudiados.
\end{abstract}

Palabras Clave - Ingeniería civil; estructuras.

Recibido: 19 de julio de 2017. Revisado: 18 de octubre de 2017. Aceptado: 27 de octubre de 2017.

\section{Reflexions about the importance and current situation of the structural area in civil engineering programs in Colombia}

\begin{abstract}
This paper, the importance of the structural area inside the core program of Civil Engineering is studied. Special characteristics of the Colombian academic programs in Civil Engineering (Structures) are taken as a case study. This country exhibit an elevated seismic hazard, and has a large stock of non-ductile buildings. In the past 35 years, moderate earth-quakes have destroyed several cities, killed thousands of people, and have produced significant infrastructure losses because an important number of its structures were designed and constructed with little or no consideration of lateral load effects (i.e. especially for those structures designed and constructed prior to the establishment of the Colombian's earthquake safety standard in 1984). In this document, three random Civil Engineering programs (Structures) in Colombia are studied, and two aspects are mainly considered: (1) professors (educational background, research production and academic syllabus), and (2) university infrastructure (labs and academic tools). It was found that the main aspect which contributes to the overall University quality is the professors' item. Therefore, several aspects related with the Colombian students inside local and international graduate programs are studied.
\end{abstract}

Keywords—-Civil engineering; structures.

\section{Introducción}

El sistema estructural, pórticos de concreto reforzado, constituye una parte importante del inventario de edificaciones en áreas propensas a sufrir de terremotos en el mundo. Un número importante de estas edificaciones, en especial las diseñadas y construidas antes de la aparición y vigencia de las provisiones sismo-resistentes, no fueron diseñadas $\mathrm{y}$ construidas para que tengan la capacidad de tener un buen desempeño ante un evento sísmico moderado o severo. Este tipo de edificaciones en especial, representan un riesgo para la integridad de los habitantes. En la Fig. 1 tomada de [1], se presenta la población mundial acumulada junto con las muertes acumuladas globales generadas por terremotos: desde 1900 a 2011, con 1000 o más muertes reportadas, y desde 1968 a 2011 para terremotos con una o más muertes. Se puede apreciar que, aun en años recientes, existe una relación directa entre el número de muertes producidas por terremotos y el incremento en la población mundial. Resulta interesante que a pesar de los avances en ingeniería sísmica, introducidos desde el final dela década de los 60's, estos avances han sido insuficientes para reducir la pendiente en la curva acumulada de muertes. Esto puede explicarse por el hecho de que el nuevo conocimiento en ingeniería sísmica es incorporado eficientemente a edificaciones nuevas, mientras que la tendencia es que el grueso de edificaciones existentes permanece sin ningún tipo de diagnóstico, evaluación y/o reforzamiento estructural. Así que, debido al crecimiento poblacional reportado en los centros urbanos, hoy por hoy, muchas más personas están expuestas a los riesgos asociados a la presencia de edificaciones construidas sin conciencia sísmica. Lo anterior es más evidente en países emergentes. Lo que confirma una de las máximas en ingeniería estructural, "Los terremotos no matan personas, las edificaciones mal diseñadas y construidas lo hacen”. Por tanto este tipo de edificaciones representan un peligro inminente para la comunidad; lamentablemente Colombia no es ajena a esta realidad.

Colombia es un país caracterizado por tener una elevada amenaza sísmica [2] acompañado de presentar una elevada vulnerabilidad sísmica de su infraestructura. En los últimos 35 años, sismos moderados (ejo; Popayán 1983 magnitud: Ms 5.0 [3], Armenia 1999 magnitud mL 6.1 [4,5]) han destruido varias ciudades, han ocasionado la muerte a cientos de personas, y han producido pérdidas significativas en su infraestructura. Un número

Como citar este artículo: Salinas-Guayacundo, D.R., Reflexiones sobre la importancia y diagnóstico del área estructural en programas de ingeniería civil en Colombia. Educación en Ingeniería, 13(25), pp. 72-81, Febrero, 2018. 


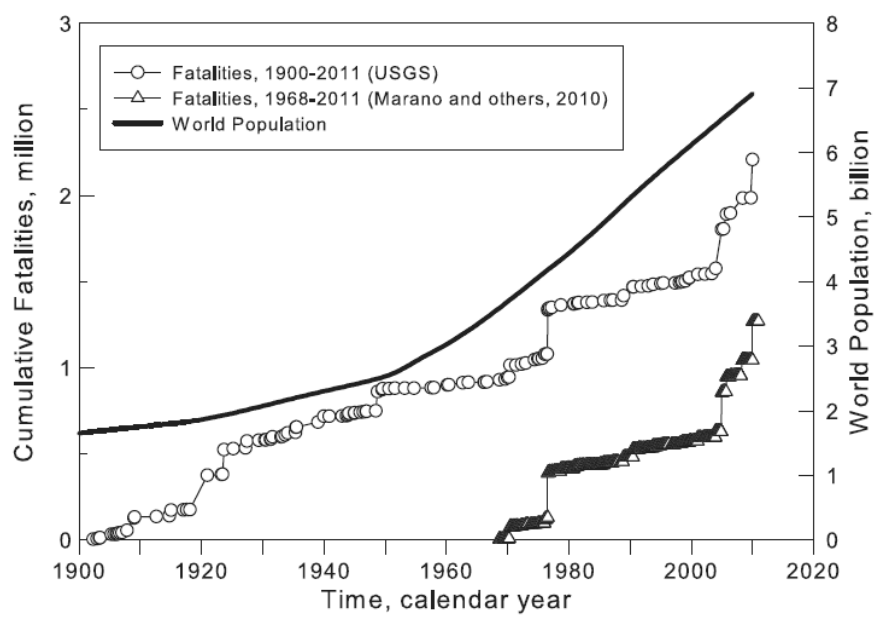

Figura 1. Muertes acumuladas globales generadas por terremotos desde $1900 \mathrm{a}$ 2011, con 1000 o más muertes reportadas, y desde 1968 a 2011 para terremotos con una o más muertes.

Fuente: [1].

importante de sus edificaciones fueron diseñadas y construidas con poca o ninguna consideración de los efectos sísmicos (especialmente en edificaciones diseñadas y construidas antes de la creación del código colombiano de diseño y construcción sismoresistente en 1984). Por otra parte, en relación a la modelación de la amenaza sísmica, se puede decir que Colombia requiere un trabajo importante en esta área, con la limitante que la Red Sismológica Nacional de Colombia (RSNC) es relativamente joven. Adicionalmente, aspectos culturales atípicos y construcción informal presentes en Colombia introducen una complejidad adicional en el proceso de determinación cuantitativa de la vulnerabilidad estructural de sus edificaciones.

Las anteriores características hacen imperativo que se fortalezca la formación de los Ingenieros Civiles en el área estructural. Se requiere una educación de calidad que incorpore los avances teóricos en este campo que permitan enfrentar competentemente las actuales necesidades del país. Lamentablemente, la realidad evidencia que la mayoría de instituciones de educación superior, donde el programa de Ingeniería Civil es ofertado, tienen un largo camino por recorrer en términos de los procesos de: acreditación, mejoramiento continuo y autorregulación de sus programas. No es admisible que, con el actual nivel de conocimiento en ingeniería estructural a nivel mundial, sismos moderados sigan produciendo elevadas pérdidas humanas y materiales. Por otra parte, resulta inconcebible que se sigan presentando, recurrentemente en los medios de comunicación, noticias evidenciando: problemas estructurales, colapsos parciales o totales en proyectos recientemente terminados o en construcción en Colombia. Por lo tanto, es pertinente revisar la importancia del área estructural dentro de los programas de pregrado en Ingeniería Civil, y mirar racionalmente su situación en el entorno académico nacional.

\section{Importancia del área estructural dentro de los programas de Ingeniería Civil en Colombia}

Colombia está localizada en el cinturón de fuego del pacifico, una de las zonas sísmicas más activas del mundo, a la cual se le atribuye el 90\% de los sismos a nivel mundial [6]. El

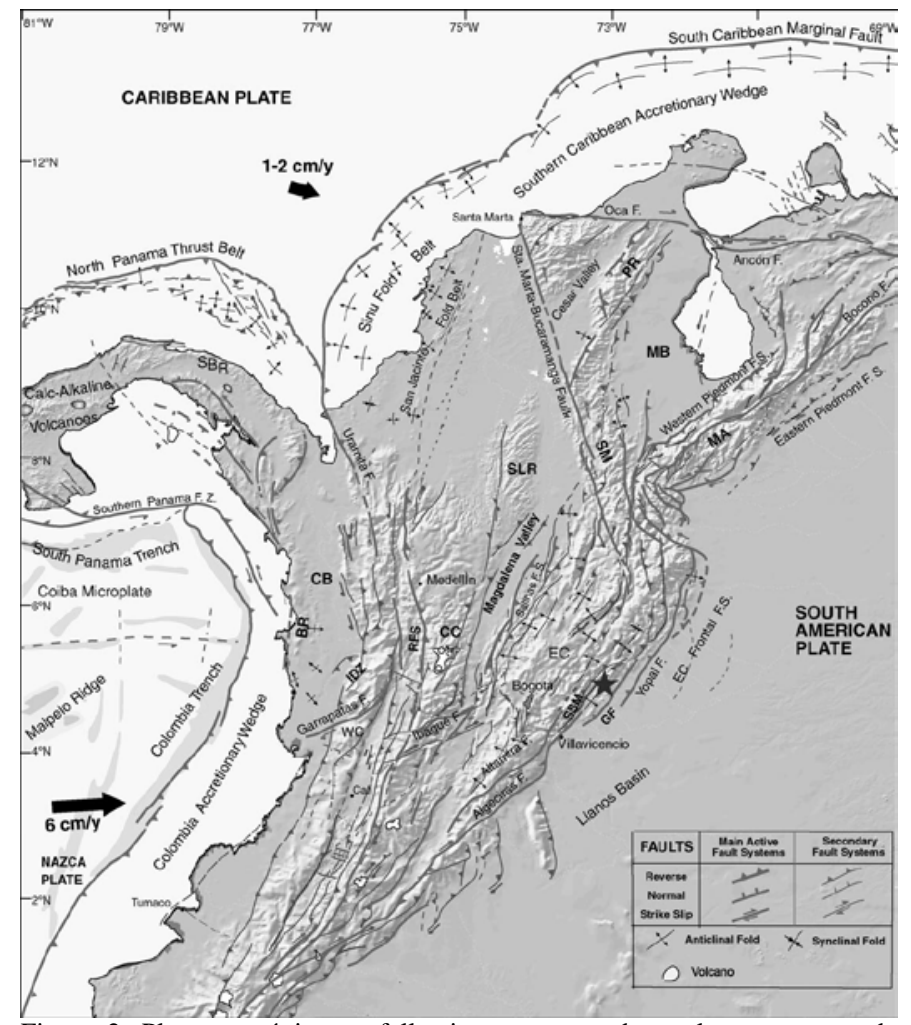

Figura 2. Placas tectónicas y fallamiento encontrado en la parte norte de Suramérica.

Fuente: [9].

territorio colombiano se encuentra ubicado sobre la placa Suramericana. En el oeste del territorio colombiano, la placa Nazca subduce debajo de la placa Suramericana a una velocidad de 61 milímetros por año, la placa Caribe se mueve en contra de la placa Suramericana a razón de $10 \mathrm{~mm}$ por año a una menor velocidad relativa [7]. Otro aspecto interesante es la presencia en Colombia del nido de Bucaramanga (The Bucaramanga nest) el cual es el lugar con mayor concentración de terremotos intermedios/profundos en el mundo [8]. La gran mayoría de habitantes en Colombia se encuentran asentados sobre la cadena montañosa de los Andes, una zona geológica de fallamiento complejo y activo mostrado en la Fig. 2 [9]. Por ejemplo, el sistema de fallas de el Romeral se extiende por más de 1600 kilómetros desde Barranquilla hasta Talara en Perú. De acuerdo con el censo nacional de población realizado en el 2005-2006 [10], y la Norma de Diseño y Construcción Sismo-Resistente de Colombia (NSR-10) [11] se puede establecer que alrededor del $90 \%$ de los Colombianos viven en zonas de amenaza sísmica alta o intermedia. Los anteriores hechos permiten establecer el elevado nivel de amenaza sísmica en el que se encuentra Colombia.

En relación a la normatividad sísmica de Colombia, el Código de Diseño y Construcción Sismo-resistente de Colombia empezó a regir por decreto en Junio 7 de 1984 como consecuencia de los terribles efectos del terremoto de Popayán, ocurrido el jueves santo, Marzo 31 de 1983 [3]. El Código colombiano se basó en el documento AIS 100 elaborado en 1981 por la Asociación Colombiana de Ingeniería Sísmica [12] el cual se fundamentaba en dos documentos: "The 
Recommended Lateral Force Requirements and Commentary” elaborado por La Asociación de Ingenieros Estructurales de California (SEAOC) escrito en 1974 [13] y el libro elaborado por el Applied Technology Council ATC 3-06 [14]. Desde su creación en 1984, el código Colombiano ha sido actualizado en dos ocasiones, en 1998 (NSR-98) y en 2010 (NSR-10). Es importante recalcar que antes de la vigencia del código de 1984, el diseño sismo-resistente no era obligatorio, adicionalmente en aquellos días existía la creencia que el diseño sismo-resistente no era requerido en edificaciones de 5 pisos o menos [15]. Lo anterior permite inferir que algunas de las edificaciones colombianas, diseñadas y construidas antes de la vigencia del código del 84 pueden carecer de la ductilidad requerida para tener un adecuado desempeño ante un evento sísmico lo que indicaría que estas edificaciones presentarían una elevada vulnerabilidad sísmica.

Sismos de mediana intensidad, han expuesto la elevada vulnerabilidad de las edificaciones colombianas. El 31 de marzo de 1983 el terremoto de Popayán afectó principalmente a la ciudad de Popayán y a los municipios del Timbío y Cajibío en el Cauca. El terremoto de magnitud Ms 5.0 produjo alrededor de 200 muertos, 2470 viviendas fueron destruidas, 6.680 sufrieron daños considerables, y aproximadamente 10.000 personas fueron damnificadas [3]. Otro claro ejemplo es el terremoto del Quindío del 25 de enero de 1999. El terremoto de magnitud mL 6.1 devastó a las ciudades de Quindío y Pereira principalmente. De acuerdo con el Servicio Geológico Americano (USGS), por lo menos 1185 personas resultaron muertas, alrededor de 700 personas se reportaron pérdidas, presuntamente muertas, se presentaron 4750 heridos, y alrededor de 250.000 personas perdieron su hogar [5]. Por otra parte, existe un estudio sobre el escenario de un sismo de magnitud 7.5 en Bogotá, [16], la conclusión convergente es que el resultado sería lamentable, en términos de pérdidas humanas y de infraestructura. Ahora bien, el estudio fue adelantado en el mayor centro urbano de Colombia, luego el panorama general sería preocupante, en especial, en lugares donde no existen procedimientos estrictos de revisión, aprobación, seguimiento y control de los proyectos de construcción. Todo lo anterior evidencia la elevada vulnerabilidad de la infraestructura Colombiana.

Por otra parte, en el campo relacionado a la caracterización de la demanda sísmica es pertinente recordar que la Red Sismológica Nacional de Colombia (RSNC) es relativamente joven. La RSNC se hizo operacional en 1993. Después de los efectos negativos del terremoto de Popayán de 1983 [3] y de la erupción del volcán nevado del Ruiz, que produjo la muerte de 20.000 personas aproximadamente en noviembre 13 de 1985, el gobierno nacional decidió invertir recursos en una nueva red sismológica [17]. Las Naciones Unidas (UN), y la Agencia Canadiense para el Desarrollo Internacional (CAID), aportaron importantes recursos económicos y de capacitación, para modernizar la RSNC que hoy es administrada por el Servicio Geológico Colombiano (antiguo INGEOMINAS) [17]. En la actualidad, la RSNC cuenta con 65 estaciones sismológicas activas, y 6 estaciones de red portátil [18]. El propósito de la red portátil es servir de apoyo a la RSNC en el registro de réplicas presentadas después de un evento sísmico fuerte, $\mathrm{y}$ también servir a los observatorios vulcanológicos en situaciones de emergencia, y también para apoyar la realización de proyectos de investigación.

Sumado a lo anterior, es pertinente tener en cuenta la realidad de la infraestructura en Colombia. En la actualidad, es común enterarse de noticias sobre edificaciones en proceso de construcción, o recién construidas, evidenciando problemas estructurales, o incluso presentando colapsos estructurales ocasionando pérdidas humanas y materiales. Dentro de las fallas más comunes aplicadas a proyectos de construcción en Ingeniería Civil se pudieran mencionar: ausencia de diseño y planeación, errores o modificaciones no autorizadas en etapas de diseño, errores en la construcción, materiales y mano de obra de baja calidad, entre otros [19]. Las anteriores causas pudieran ser detectadas si un proceso competente de revisión, supervisión y control es estrictamente aplicado a los proyectos de infraestructura en Colombia. Lamentablemente, la calidad y eficiencia en los procesos de seguimiento y control varía considerablemente dependiendo de la localización del proyecto, y las entidades encargadas de revisar, aprobar los diseños y supervisar la construcción del mismo. La importancia en la revisión en las etapas de diseño y construcción resulta evidente en sistemas estructurales construidos en concreto reforzado, por mencionar un ejemplo. En este tipo de sistemas estructurales, el detallamiento, es decir la colocación, dimensiones y especificaciones del acero de refuerzo y concreto, controla la resistencia del sistema estructural.

Por lo anteriormente expresado, es innegable la elevada amenaza y vulnerabilidad sísmica presentada en Colombia. En la actualidad, existe un elevado porcentaje de edificaciones vulnerables a presentar colapso parcial o total en Colombia ante un evento sísmico mayor o moderado. A pesar de que las edificaciones diseñadas únicamente para resistir acciones gravitacionales tienen cierta resistencia lateral inherente, esta resistencia no es suficiente para que tengan un buen desempeño ante un evento sísmico [6]. Lo anterior ha sido validado en la reciente historia sísmica en Colombia [3-5]. Aunque en los últimos años, algunas edificaciones han sido reforzadas estructuralmente en Colombia, el grueso de sus edificaciones no presenta intervención alguna. Lo anterior en parte, por la falta de políticas gubernamentales, falta de interés y conciencia sísmica de los propietarios, pero en especial, por la ausencia en la investigación, propuesta, validación e implementación de técnicas factibles, eficientes y prácticas aplicadas al reforzamiento estructural de edificaciones en Colombia. Luego, las Universidades y centros de investigación tienen una responsabilidad importante en este sentido. En la actualidad, es común apreciar que las personas que viven en edificaciones con elevada vulnerabilidad, a menudo, no son conscientes de la elevada vulnerabilidad de sus hogares. Lo anterior puede ser explicado por la historia sísmica reciente en Colombia donde, si bien en cierto, se han presentado algunos eventos sísmicos, estos eventos han sido de magnitud moderada/baja, alejados de los centros urbanos, o estos se han presentado a una profundidad considerable, donde a pesar de ser percibidos por los habitantes, el efecto sobre las edificaciones no es de importancia. Lo anterior ayuda a afianzar el falso nivel de seguridad percibido por los propietarios de estas edificaciones y de la comunidad en 
general, al mismo tiempo desestimula la formulación de proyectos de investigación y reforzamiento estructural; y se relega a un segundo plano, la importancia del área estructural en programas de Ingeniería Civil.

Por lo tanto, es necesario que los programas académicos de Ingeniería Civil, en el área estructural, respondan de una manera eficaz ante las problemáticas anteriormente presentadas. Bajo el criterio del autor, y basado en el elevado riesgo y vulnerabilidad de la infraestructura en Colombia, se requiere que dentro del área estructural se incorporen los avances en esta área del conocimiento y específicamente que se profundice en el estudio de la: (1) modelación determinística y probabilística del riesgo sísmico, apoyados en los datos aplicables al caso Colombiano suministrados por la RSNC, (2) modelación sistemática y cuantitativa que permitan determinar la vulnerabilidad de diversos sistemas estructurales, (3) propuesta, modelación, cuantificación y validación de técnicas eficaces de reforzamiento estructural, y (4) formación integral de profesionales competentes en la elaboración, revisión, y construcción de proyectos de infraestructura.

Una vez presentada la justificación conceptual sobre la importancia del área Estructural dentro de los programas de formación en Ingeniería Civil en Colombia, se procede a revisar su situación actual, tendencias y prácticas que se encuentran en algunas universidades en Colombia.

\section{Análisis de la situación actual del área estructural en tres programas de Ingeniería Civil en Colombia}

El objetivo de la presente sección es revisar la situación actual del área estructural en algunos programas de Ingeniería Civil ofrecidos en Colombia, a partir de la comparación directa de tres programas académicos seleccionados al azar, de entre un ranking de programas de Ingeniería. La idea de seleccionar programas académicos de un ranking universitario existente en Colombia, tiene que ver con la posibilidad de comparar únicamente tres programas académicos que permitan emitir unas reflexiones preliminares sobre la situación actual del área estructural en programas de Ingeniería Civil en Colombia, sin la necesidad de realizar un análisis detallado sobre una muestra representativa de las instituciones colombianas. El objetivo es seleccionar el primer programa académico buscando que se encuentre ubicado en los primeros lugares del ranking, el segundo y tercer programa académicos se esperan se encuentren en una posición aceptable y promedio, respectivamente, dentro del ranking. En ese sentido, se seleccionó el ranking elaborado por la revista dinero [20], quien utilizó los resultados de las pruebas Saber Pro de 2016 para elaborar un ranking de las Universidades Colombianas agrupados según los pregrados que ofrecen. De este ranking, únicamente los resultados presentados bajo el nombre de "ingeniería” son usados en este documento. El grupo “ingeniería” agrupa los siguientes programas de ingeniería: sistemas, industrial, electrónica, civil, mecánica, ambiental, eléctrica, de alimentos, agroindustrial, agronómica y química. Es pertinente recalcar que las pruebas Saber Pro evalúan tanto las competencias específicas de cada programa así como las competencias genéricas (comunicación escrita, razonamiento cuantitativo, lectura crítica, competencias ciudadanas e inglés). En la Fig. 3 se presenta el resultado de esta clasificación, agrupada según la localización de la geográfica de la Universidad. En la Fig. 3, el color de cada círculo indica el departamento donde se encuentra ubicada la institución universitaria, mientras que el tamaño del circulo es un indicativo de la posición de la Universidad dentro del ranking; es decir, círculos grandes representan los primeros lugares y a medida que se reduce el tamaño del círculo, indica puestos inferiores dentro del ranking. En el eje vertical de la gráfica, se presentan únicamente las 150 mejores universidades en ingeniería según la clasificación reportada por la revista dinero. Ahora, antes de proceder a seleccionar y analizar los tres programas académicos, es conveniente analizar la información contenida en la Fig. 3. De la gráfica se observa tanto como en Antioquia y Cundinamarca, la oferta académica es amplia y diversa; la percepción implícita de calidad, es decir su posicionamiento dentro del ranking, varia de forma gradual. Por otra parte, en las universidades localizadas en Atlántico, Bolívar, Caldas y Santander, se presentan marcadas transiciones entre dos instituciones educativas; con diferencias aproximadas de 70, 50, 50 y 60 puestos respectivamente, entre dos universidades del mismo departamento. En Boyacá, una misma institución, con tres seccionales localizadas en diferentes ciudades, obtuvo los mejores resultados, con una diferencia aproximada de 15 puestos entre las seccionales. Esta institución marcó una diferencia significativa entre las demás Universidades del departamento, aproximadamente 30, 60 y 100 puestos. Finalmente respecto a las universidades localizadas en el departamento del Valle del Cauca, se puede afirmar que aproximadamente el $70 \%$ de sus universidades se localiza dentro de los mejores 65 puestos del ranking de los programas de Ingeniería analizados, lo que entrega una percepción de homogeneidad en las mayoría de sus instituciones.

Es pertinente aclarar que si bien es cierto, se pudiera argumentar que existiría cierta subjetividad al clasificar una institución de educación superior únicamente por medio de un parámetro discreto, que en este caso, es el resultado de la prueba Saber Pro de 2016, el ranking seleccionado tiene como finalidad proveer un listado jerárquico sobre el cual se seleccionaran tres programas académicos al azar, con las características ya mencionadas, para analizar la situación actual del área estructural dentro del programa de Ingeniería Civil es estas Universidades.

Luego, la anterior información brinda un contexto adecuado para estudiar tres instituciones localizadas dentro de las 70 primeras instituciones del ranking seleccionado. Principalmente, cada universidad es estudiada teniendo en cuenta dos enfoques: (1) el talento humano asociado al área estructural, es decir, formación de los docentes, productividad académica, y pertinencia de los contenidos programáticos, y (2) la infraestructura de la Facultad, es decir, laboratorios, y equipos usados en el proceso de enseñanza. Los datos para realizar el análisis de los programas académicos fueron extraídos directamente de las páginas web de estas instituciones. Se filtró el ranking y para introducir cierta homogeneidad, al respecto, únicamente programas académicos de Ingeniería Civil, que poseen acreditación entregada por el 


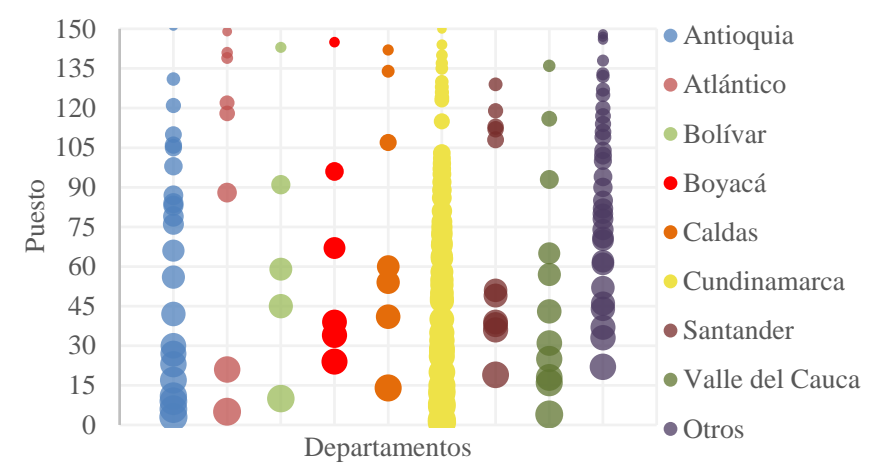

Figura 3. Gráfico de burbujas ranking de los 150 primeros puestos por departamento, según [20].

Fuente: El Autor.

CNA (Consejo Nacional De Acreditación) fueron considerados. En conveniente aclarar que, en términos generales, el proceso de acreditación universitaria en Colombia, es un proceso institucional voluntario a través del cual se busca que las instituciones garanticen a la sociedad un adecuado nivel de calidad y credibilidad en el quehacer docente. A continuación se presentan las instituciones de educación superior seleccionadas aleatoriamente.

\subsection{Universidad 1}

En el ranking En el ranking [20], esta institución se encuentra ubicada dentro de los primeros 10 puestos. Según los datos provistos en la página web oficial de esta institución, Los docentes que laboran en el área estructural del programa de Ingeniería Civil, cuatro (4) poseen formación doctoral y dos (2) tienen el título maestría como formación máxima obtenida, ambas titulaciones obtenidas en Universidades Americanas, y un doctorado obtenido en una Universidad Británica. Adicionalmente, existen dos (2) docentes con formación de magister en el área estructural, titulaciones obtenidas una en Colombia, y otra en el exterior. Todos los títulos universitarios fueron obtenidos en el área estructural. Respecto a la productividad investigativa, los docentes del área estructural cuentan con más de 50 artículos de investigación publicados en revistas catalogadas dentro del cuartil 1 (Q1) en SJR (SCImago journal Rank), además, cuentan con un número importante de participaciones en eventos nacionales e internacionales. A pesar, de que el Programa de Ingeniería Civil es de 4 años, los contenidos del área estructural son cubiertos adecuadamente e incorporan un adecuado balance entre los métodos tradicionales de análisis estructural y los métodos matriciales. Los laboratorios del área estructural son de última tecnología; además, los docentes cuentan con un número importante de modelos a escala reducida para apoyarse en la enseñanza teórica y conceptual de temas relacionados con Ingeniería Estructural.

\subsection{Universidad 2}

Esta institución se encuentra ubicada dentro del puesto (30 al 40) según [20]. Basados en los datos reportados en la página oficial de esta institución universitaria, en el programa de Ingeniería Civil, el área estructural cuenta con cinco (5) docentes titulares asignados al área estructural, cuatro (4) de ellos poseen título de magíster en Ingeniería Civil, de los cuales tres (3) lo obtuvieron en Colombia, y uno (1) lo obtuvo en una Universidad de Latinoamérica. El docente restante, posee título de especialista obtenido en una institución en Colombia. Los cincos (5) docentes poseen sus estudios de posgrado en el área específica del conocimiento, es decir formación en estructuras. Respecto a la productividad académica, no aparecen registrados productos de investigación en revistas internacionales, sin embargo se relacionan algunas capacitaciones en cursos de corta duración. La Facultad cuenta con un laboratorio modesto relacionado con el área estructural, no se reconoce la existencia de modelos a escala usados en la enseñanza de las asignaturas del área estructural. El programa académico es de 5 años, y en relación a los contenidos programáticos, se debe decir que estos son pertinentes. Adscrito a la Facultad de Ingeniería Civil, se encuentra asociado un programa de Especialización es Estructuras.

\subsection{Universidad 3}

Esta institución se encuentra ubicada dentro del puesto (50 al 70) de acuerdo con [20]. Según los datos proporcionados en la página web oficial de esta institución, se relaciona nueve (9) docentes adscritos al área estructural. Cuenta con tres (3) docentes con título de especialista y un (1) docente con título de maestría, ambas titulaciones obtenidas en el área estructural. Tres (3) docentes poseen títulos de especialización y uno (1) adelanta estudios de maestría, ambos en otras áreas del conocimiento. El docente restante, no es ingeniero Civil, pero posee título doctoral en otra área del conocimiento diferente al área estructural, dicho posgrado fue realizado en Colombia. Respecto a la productividad académica, se registran dos artículos internacionales, y seis nacionales, ambas producciones en un área diferente al área estructural. La Facultad no registra laboratorio asociado con el área estructural; al igual no se reconoce la existencia de modelos a escala usados en la enseñanza de las asignaturas del área estructural. El programa de Ingeniería Civil es de 5 años y respecto a los contenidos programáticos se puede decir que se hace demasiado énfasis en métodos tradicionales de análisis y los métodos matriciales de análisis estructural, tienden a ser ignorados. Otros contenidos son suficientes, pero al ser orientados por docentes con formación profesional en otra área del conocimiento, se desconoce el enfoque, tratamiento y profundidad con que se imparten. Existe un programa de Especialización en Estructuras adscrito a la Facultad de Ingeniería Civil en esta universidad, pero este programa cuenta con docentes externos diferentes, en su mayoría, a los vinculados al área de estructuras de pregrado.

\subsection{Análisis de las universidades presentadas}

Si bien es cierto existe una diferencia significativa en términos de infraestructura entre las Universidades analizadas en este documento, se puede establecer que la diferencia esencial tiene que ver con el recurso humano. La Tabla 1 presenta la relación 
Tabla 1

Porcentaje de docentes según formación profesional y área del conocimiento en las Universidades analizadas.

\begin{tabular}{ccccccc}
\hline \multirow{2}{*}{ Universidad } & \multicolumn{2}{c}{$\begin{array}{c}\text { Especialistas } \\
\mathbf{( \% )}\end{array}$} & \multicolumn{2}{c}{ Magister (\%) } & \multicolumn{2}{c}{ Doctorado (\%) } \\
\cline { 2 - 7 } & Estructuras & Otras & Estructuras & Otras & Estructuras & Otras \\
\hline $\mathbf{1}$ & 0 & 0 & 50 & 0 & 50 & 0 \\
\hline $\mathbf{2}$ & 20 & 0 & 80 & 0 & 0 & 0 \\
\hline $\mathbf{3}$ & 33.3 & $\mathbf{4 4 . 4}$ & 11.1 & 0.0 & 0.0 & $\underline{\mathbf{1 1 . 1}}$ \\
\hline
\end{tabular}

Fuente: El autor.

porcentual asociada a la formación profesional de los docentes de las tres universidades analizadas. Es claro que la universidad 3 posee un elevado porcentaje de sus docentes con formación como especialistas (78\%), pero lo más llamativo es que el 56\% de sus docentes tienen formación académica en un área diferente al área estructural, a pesar de la importancia de esta área y responsabilidad dentro de los programas de formación en Ingeniería Civil discutidos en el literal 2 de este documento. Adicionalmente, resulta evidente el bajo nivel de docentes con formación doctoral en estructuras en las tres universidades analizadas. La universidad 1 cuenta con docentes adscritos al área estructural con formación doctoral en esta área del conocimiento y registra un $50 \%$ de sus docentes con esta formación. Las universidades 2 y 3 no poseen docentes con formación doctoral en estructuras. A pesar de que la universidad 3 cuenta con un docente con formación doctoral, al no tener formación previa como Ingeniero Civil y al registrar maestría y doctorado en un área del conocimiento diferente al área estructural, se desconoce la contribución y el impacto dentro del área estructural en esta institución.

Por lo tanto, resulta evidente la importancia del factor humano dentro de las universidades. La importancia del área estructural en programas de Ingeniería Civil en Colombia, requiere profesionales comprometidos y con formación de alto nivel para adelantar los procesos de investigación, desarrollo e innovación que este país requiere. Lo anterior siendo consistente con la elevada amenaza y vulnerabilidad sísmica de la infraestructura de este país. La Tabla 1 es clara en indicar el bajo porcentaje de docentes con formación doctoral en estructuras dentro de los programas de Ingeniería Civil analizados. Por lo anterior, es conveniente dar una mirada sobre la formación doctoral en programas de Ingeniería Civil en Colombia para poder entender las tendencias actuales y prácticas comunes que ayuden a explicar déficit de docentes con formación doctoral de calidad en el área estructural.

\section{Antecedentes sobre la formación doctoral en Ingeniería en Colombia}

En la década del 80 en Colombia, el Departamento Administrativo de Ciencia, Tecnología e Innovación de Colombia (Colciencias) incluye en su presupuesto recursos encaminados a la formación de recurso humano de alto nivel [21]. Colombia inicia tarde este proceso comparado, con países como Estados Unidos, y México que desde 1945 y 1970, respectivamente ya impulsaban programas de formación doctoral. En la actualidad, se estima que Colombia gradúa 6.6 doctores por cada millón de habitantes ( $6.6 \mathrm{~d} / \mathrm{mh})$. En contraste,
Estados Unidos gradúa alrededor de $200(\mathrm{~d} / \mathrm{mh})$, la India, un país emergente, gradúa alrededor de $20(\mathrm{~d} / \mathrm{mh})$ [22], y en Latinoamérica se tiende a triplicar la cifra colombiana. Según cifras de Colciencias, entre 2004 y 2014 se han graduado 2.636 que han cursado, al menos uno, de los más de 205 programas que ofrecen las universidades locales. Según el CNA, a 2014 en Colombia existen alrededor de 43 instituciones académicas que ofrecen programas de formación doctoral, muy pocos de estos programas se encuentran acreditados y se requerirá un tiempo prudente para que la calidad de los mismos y el impacto de sus egresados sean evaluados con miras a su acreditación. Es pertinente aclarar que, al igual que sucede en los programas de pregrado, el proceso de acreditación de los programas de posgrado, es un proceso autónomo y voluntario del programa doctoral. El proceso de acreditación se fundamenta en políticas de autoevaluación, mejoramiento continuo y autorregulación, a diferencia de los procesos de registro calificado, el cual es un proceso obligatorio. Este proceso toma varios años llevarlo a cabo, y en caso de no obtenerse los resultados esperados se puede (i) tratar de aplicar medidas correctivas o (ii) simplemente requerir o tramitar un nuevo registro calificado y empezar el proceso de nuevo. Por lo anterior la oferta académica de programas doctorales internacionales, que cuentan con una adecuada trayectoria, calidad y respaldo académico e investigativo, resulta más atractiva y confiable desde el punto de vista académico, puesto que permite disminuir la incertidumbre asociada a la calidad, comparado con los programas doctorales nacionales que aún no se encuentran acreditados. Al mismo tiempo, según el SCImago Institutions Rankings (SIR) 2017, tres Universidades colombianas se ubican dentro de los primeros 600 puestos (se ubican los puestos 557, 593, 597) dentro del ranking mundial de investigación. Luego, si se cuenta con los recursos de financiación suficientes, los anteriores argumentos pueden influenciar la escogencia de realizar un programa doctoral internacional.

En Colombia en las últimas décadas ha aumentado considerablemente la demanda de doctores. Las motivaciones para realizar un doctorado se fundamentan en el deseo de mejorar las capacidades técnicas, analíticas, críticas y conceptuales, mejorar competitividad, desarrollar las competencias de trabajo autónomo encaminadas a la construcción de nuevo conocimiento que aporte al desarrollo de la comunidad. Adicionalmente, en Colombia la política de Ciencia, Tecnología e Innovación (CTI) es uno de los principales lineamientos del Plan Nacional de Desarrollo 20142018 [23], su ideal es lograr una Colombia en paz, equitativa y la más educada de América Latina. En el diagnóstico de la política del CTI se identificó, principalmente, que el país y sus regiones no han logrado impulsar el desarrollo económico y social a través de la Ciencia, Tecnología e Innovación, por lo tanto resulta imperativo invertir, en el mediano plazo, en el capital humano pensando en el fortalecimiento futuro de: (1) investigación y desarrollo, (2) innovación y emprendimiento, y (3) transferencia de conocimiento y tecnología. Producto de lo anterior en los últimos años, el gobierno nacional, a través de Colciencias en especial, ha trabajado principalmente en apoyar la realización de estudios doctorales en instituciones 
académicas reconocidas, dentro y fuera del país. La decisión de optar por una formación doctoral en Colombia, o en el exterior radica principalmente en las restricciones de: aceptación, económicas, familiares, y de segunda lengua. Se estima que en promedio un doctorado nacional puede valer $\$ 250$ millones y uno en el exterior, alrededor de $\$ 400$ millones [24], sin incluir, en programas de tiempo completo, el costo asociado al lucro cesante que el estudiante experimenta en su etapa de estudios. Recientemente, se ha presentado una desaceleración importante de colombianos accediendo a estudios doctorales nacionales e internacionales. Según cifras de Colciencias las becas para doctorado pasaron de 650 en 2015 a 222 en 2016. La desaceleración puede atribuirse principalmente la fuerte reevaluación que el dólar ha registrado respecto a la moneda local en los últimos años. Cabe recalcar que, la vasta mayoría de profesionales en Colombia requieren de becas para poder acceder a la oferta doctoral. Ahora bien, estas becas son, en realidad, créditos condonables, que como cualquier otro préstamo generan intereses, pero si se termina satisfactoriamente el doctorado, se retorna al país y se trabaja un periodo de tiempo en actividades de CTI, se puede aplicar a un porcentaje de condonación de la deuda, y el capital restante no condonado, más los intereses generados, deberán ser cancelados por el beneficiario en unas condiciones predefinidas. Entidades como Colfuturo, en su programa genérico de crédito beca, ofrecen créditos-becas condonables que van del 50 al $60 \%$ del capital entregado, y programas de Colciencias pueden cubrir hasta el 100\% del capital prestado.

\subsection{Situación actual de los programas doctorales en Ingeniería Civil en Colombia}

Es necesario puntualizar que en Colombia a nivel de pregrado, En el 2015, existían cerca de 6.400 programas de educación superior de los cuales 909 (14,2\%) estaban acreditados, y en términos de universidades, de un total de 287 instituciones de educación superior 39 (13,6\%) contaban con acreditación institucional [22]. Ahora en lo referente a programas de posgrado en Ingeniería Civil, se debe mencionar que existe una baja oferta de programas de Maestría y Doctorado. Un elevado porcentaje de los mismos, se concentran en pocas instituciones de educación superior ubicadas principalmente en Bogotá, Medellín, Cali y Bucaramanga. Lamentablemente, la oferta académica de calidad nacional no ha crecido tan rápido como la demanda, por lo anterior se ha observado dos (2) tendencias en los últimos años aplicables a los profesionales formados como Ingenieros Civiles en Colombia, específicamente se ha observado que estos profesionales: (i) incursionan en programas doctorales diferentes al área de desempeño y experticia previa, y (ii) acceden a programas doctorales online o semi-presenciales, de discutible calidad y rigurosidad académica.

Como se mencionó anteriormente, uno de las mayores limitantes en la formación doctoral en Ingeniería Civil y en otras áreas del conocimiento, es la centralización y la baja oferta académica comparada con la demanda actual. Como consecuencia de lo anterior, en los últimos años se ha vuelto más común ver ingenieros accediendo a ofertas doctorales diferentes a su perfil académico. Por ejemplo, es el caso de Ingenieros Civiles, desempeñándose en áreas como: Estructuras, Geotecnia, Recursos Hídricos, Ambiental, Vías y Transporte y Construcción, realizando doctorados en áreas diferentes al área de experticia previa, generalmente accediendo a Doctorados en Educación, u otros programas doctorales ofertados cerca de la ciudad de trabajo y residencia del profesional. Esta opción le permite a docentes que ya se encuentran vinculados laboralmente a una institución de educación superior a que exploren un área nueva del conocimiento, y de una manera flexible, puedan estudiar y seguir trabajando simultáneamente. Finalizado el programa doctoral, el docente podrá acceder a un ascenso en su escalafón docente y mejorar su nivel salarial.

Las modalidades de doctorados online o semi-presencial han cobrado mucha fuerza en los últimos años en Colombia. Los elevados costos de la oferta académica de programas doctorales de calidad internacionales y nacionales, sumados a la baja tasa de retorno de la inversión en el mediano plazo, justificada con la baja remuneración salarial presentada en la mayoría de las universidades colombianas, han contribuido a que esta opción académica se fortalezca. Los profesionales que optan por esta opción lo hacen principalmente por diversas razones entre las que se tienen: (1) se puede seguir trabajando, sin necesidad de requerir licencia de estudios, (2) la aceptación, evaluación, y estudios son considerablemente más flexibles, (3) se tiene una mínima disrupción en la vida cotidiana, en especial, en lo relacionado al ámbito personal, familiar y profesional, (4) se tiene un costo significativamente menor, comparado con las opciones presenciales nacionales o internacionales. Al mismo tiempo, la relación costo-beneficio es más atractiva, puesto que se involucra una inversión menor, y los beneficios salariales son idénticos comparados con las opciones presenciales nacionales e internacionales de calidad. Lo anterior siempre y cuando el diploma sea convalidado, en caso de que el diploma provenga del exterior. Lo anterior, permitirá encontrar el punto de equilibrio de la inversión en un menor tiempo, (5) baja tasa de acceso a la oferta presencial nacional e internacional, y (6) presunción de titulación universal; al respecto, en Colombia en especial, es común que los egresados de programas doctorales de todo tipo, se autodenominen con el mismo título "Ph.D" y que esta información aparezca consignada en los perfiles académicos en algunas de las páginas web oficiales de las universidades colombianas. Con lo anterior, faltando a la verdad puesto que exhiben a la comunidad académica y público en general una titulación, de reconocida valía y respeto internacional ("Ph.D”), titulación que en la mayoría de casos, no les ha sido conferida.

Referente a lo anterior, es conveniente precisar que por definición universal, el título de cualquier profesional corresponde al título que le fue conferido por la universidad y que se encuentra consignado en el diploma o acta de grado respectiva. Uno de los títulos más prestigiosos y de reconocimiento es el de Doctor of Philosophy, conocido en siglas como "Ph.D" o " $\mathrm{PhD}$ ", es un tipo de doctorado entregado principalmente por el sistema universitario anglosajón y el cual se puede obtener en diversas áreas del conocimiento, después de cumplir con una rigurosidad académica e investigativa, que se traducen el aporte innovador al 
conocimiento. En la actualidad, no se entienden claramente las razones que justifiquen el por qué cientos de profesionales radicados en Colombia, que (i) adelantaron sus estudios doctorales (en Colombia o Latinoamérica, mediante modalidad presencial, semi-presencial, o virtual), (ii) se les confirió un diploma diferente, por ejemplo, Doctor en Educación, Doctor en Historia, Doctor en Derecho, Doctor en Ingeniería, Doctor en Teología, etc.), y (iii) sus estudios se adelantaron por medio de seminarios, conferencias, y sistemas laxos de evaluación, impartidos en idioma español; todos los anteriores, terminen atribuyéndose otra titulación, y en otro idioma "Doctor of Philosophy”, específicamente "Ph.D”. Lo cierto es que las ofertas académicas de baja calidad se han vuelto tan comunes, que incluso personal administrativo de las universidades han optado por obtener este tipo de titulación doctoral para mejorar sus perspectivas laborales y salariales. Por otra parte, a pesar que el usar incorrectamente o inapropiadamente una titulación que no se ha obtenido va en contra de la ley Colombiana [25,26], este tipo de errores son cada vez más recurrentes en las páginas oficiales de las universidades, eventos académicos, administrativos y culturales sin que se evidencie un control o regulación eficiente al respecto en Colombia. Todo esto contribuye a devaluar y popularizar el título de "Ph.D", un título académico de marcado reconocimiento y prestigio al mismo tiempo que estimula la escogencia del “atajo académico”.

Tabla 2.

Comparación programa de doctoral acreditado internacional versus programa semi-presencial online internacional.

\begin{tabular}{|c|c|c|}
\hline Característica/titulación & Ph.D & Doctorado \\
\hline Modalidad & Presencial & $\begin{array}{c}\text { Flexible (semi- } \\
\text { presencial, online) }\end{array}$ \\
\hline Fundación Universidad & Mayor a 100 años & Menor a 15 años. \\
\hline Ranking nacional & $\begin{array}{c}\text { Entre los mejores } \\
10 \\
\end{array}$ & $\begin{array}{l}\text { No registra ranking } \\
\text { nacional, ni estatal. }\end{array}$ \\
\hline \multirow{3}{*}{ Requisitos de ingreso } & Hoja de vida & Hoja de vida \\
\hline & $\begin{array}{c}\text { Exámenes de } \\
\text { Ingreso (GRE, } \\
\text { TOEFL) }\end{array}$ & - \\
\hline & $\begin{array}{c}\text { Ensayo de } \\
\text { candidatura }\end{array}$ & - \\
\hline \multirow{4}{*}{ Requisitos de grado } & $\begin{array}{l}\text { Aprobar cursos } \\
\text { tomados }\end{array}$ & $\begin{array}{l}\text { Realizar trabajos } \\
\text { asignados. }\end{array}$ \\
\hline & $\begin{array}{l}\text { Aprobar el examen } \\
\text { de clasificación }\end{array}$ & $\begin{array}{l}\text { Asistencia a los } \\
\text { seminarios. }\end{array}$ \\
\hline & $\begin{array}{l}\text { Aprobar el examen } \\
\text { de Candidatura }\end{array}$ & $\begin{array}{c}\text { Realizar trabajo de } \\
\text { grado. }\end{array}$ \\
\hline & $\begin{array}{l}\text { Aprobar el examen } \\
\text { final (disertación) }\end{array}$ & \\
\hline \multirow[t]{2}{*}{ Duración } & \multirow{2}{*}{$\begin{array}{c}\text { Incierta (aprox. } 5 \\
\text { años) }\end{array}$} & $\begin{array}{c}2 \text { años si se tiene } \\
\text { maestría }\end{array}$ \\
\hline & & 3 años sin maestría \\
\hline $\begin{array}{c}\text { Disrupción de la vida } \\
\text { cotidiana durante estudios }\end{array}$ & Total & mínima \\
\hline Costo relativo a un $\mathrm{PhD}$ & $100 \%$ & $(5-10) \%$ \\
\hline $\begin{array}{l}\text { Esfuerzo requerido } \\
\text { (max,min) }\end{array}$ & $(100,80) \%$ & $(10,5) \%$ \\
\hline $\begin{array}{c}\text { Puntos a recibir en } \\
\text { convocatorias docente } \\
\text { (max,min) }\end{array}$ & $(30,20) \%$ & $(30,20) \%$ \\
\hline $\begin{array}{l}\text { Existe diferencia en } \\
\text { asignación salarial? }\end{array}$ & No & No \\
\hline $\begin{array}{l}\text { Convalidación aprobada en } \\
\text { Colombia? }\end{array}$ & Si & $\mathrm{Si}$ \\
\hline
\end{tabular}

Fuente: El autor.
A manera de ilustración, la Tabla 2 presenta un paralelo entre un programa doctoral internacional que otorga el título de Doctor of Philosophy (Ph.D) y un programa semi-presencial, online ofertado en un país Latinoamericano. Los programas se seleccionaron con el criterio de contar con egresados de ambos programas con resolución aprobada de convalidación otorgada por el Ministerio de Educación Nacional de Colombia. Es preciso aclarar que, el proceso de convalidación es un trámite administrativo por el cual se reconoce en Colombia titulaciones obtenidas en el exterior mediante un acto administrativo que expresa su equivalencia en el territorio colombiano. Al analizar la Tabla 2, resulta evidente la diferencia significativa en términos de procesos de admisión, exigencia, respaldo institucional, seriedad, costo económico, y sacrificio; sin embargo, para efectos prácticos, ambos egresados recibirán el mismo puntaje, por concepto de formación académica para efectos salariales en cualquier universidad en Colombia, sin mencionar, el hecho de que los egresados de programas semipresenciales online internacional y otros continúen autodenominándose imprecisamente en Colombia como "Ph.D”.

Es pertinente aclarar que un egresado de un programa doctoral de calidad nacional o internacional tuvo principalmente que: (1) incurrir en un préstamo importante de recursos económicos (entre 250 y 500 millones de pesos, aproximadamente [24]) y asumir los intereses generados por este capital desde su desembolso hasta el momento en que se realice el pago, (2) dedicarse de tiempo completo a este objetivo, por lo que en la mayoría de casos, involucró el no laborar durante el periodo de estudios, (3) enfrentar la incertidumbre sobre la culminación exitosa del programa dentro del plazo programado, y manejar niveles elevados de presión y frustración en el proceso de construir nuevo conocimiento, (4) desempeñarse competentemente en un entorno altamente competitivo y globalizado, (5) descuidar temas familiares, personales, de salud, para llevar a buen término el objetivo trazado y (6) renunciar a posibilidades laborales en el exterior, con el fin de responder con los compromisos previamente adquiridos y aceptados con el(los) otorgante(s) del préstamo. Luego no existe coherencia en el trato igualitario en las condiciones, comparado con los profesionales que han realizado estudios online o semi-presenciales nacionales o internacionales y todo lo anterior ayuda a explicar el déficit de profesionales en el área estructural en programas de Ingeniería Civil.

Teniendo en cuenta todo lo expresado anteriormente, la combinación de demanda centralizada, baja oferta de programas de calidad nacionales en el área específica del conocimiento, costos elevados de las ofertas académicas de calidad nacionales e internacionales, condiciones de inequidad, falta de regulación por parte de las entidades competentes, entre otros, han contribuido a que profesionales con formación en Ingeniería Civil se estanquen en sus áreas de especialidad o cambien de área de conocimiento o simplemente accedan a ofertas académicas de baja calidad y poca rigurosidad académica accediendo a la creciente oferta online o semipresencial provenientes, principalmente, de países hispanohablantes. A través de este documento se ha demostrado 
que el área estructural tiene, no solo un papel vital dentro del programa académico en Ingeniería Civil, sino esta área del conocimiento tiene una responsabilidad sustancial con la comunidad puesto que de esta dependen vidas humanas, a igual que la seguridad e integridad de las ciudades. Por lo tanto, en un país con una elevada amenaza y vulnerabilidad como Colombia, no se puede delegar su instrucción a docentes con formación académica diferente al área estructural y se debe estimular la formación doctoral en programas de alto nivel nacionales e internacionales para que mediante la investigación, desarrollo e innovación formulen propuestas eficaces que contribuyan a mitigar los devastadores efectos de sismos en la infraestructura Colombiana en el futuro.

\section{Conclusiones}

En un programa de Ingeniería Civil, el área estructural es de vital importancia, de ella dependen vidas humanas, al mismo tiempo, con una aplicación competente en esta área se pueden salvar vidas. Colombia es un país que exhibe un elevado riesgo sísmico y posee una vulnerabilidad sísmica alta de sus edificaciones. Se estima que el $90 \%$ de sus habitantes viven en zonas de amenaza sísmica alta o intermedia, lamentablemente su infraestructura no es consecuente con esta realidad. Un porcentaje importante de las edificaciones colombianas requieren algún tipo de intervención estructural para garantizar un adecuado desempeño en un evento sísmico. Por lo anterior se requiere que se entienda la importancia del área estructural y no se delegue irresponsablemente la enseñanza de esta área a profesionales especializados en otras áreas del conocimiento. Por otra parte, es necesario que desde las universidades, profesionales con formación en el área estructural propongan, evalúen y validen estrategias eficientes de reforzamiento estructural, consistentes con la adecuada representación de la amenaza sísmica y que puedan ayudar a disminuir la pérdida potencial de vidas humanas ante un eventual movimiento sísmico en el futuro.

El ranking de programas de ingeniería en Colombia usado en este documento sirvió para analizar discretamente la situación del área estructural en tres (3) programas de Ingeniería Civil que cuentan con acreditación otorgada por parte del CNA y que ocupan diferentes posiciones dentro del ranking seleccionado en este documento. Se identificó que el factor más relevante dentro de estos programas es el factor humano. Se evidenció que existe un bajo porcentaje de docentes con formación doctoral en el área estructural en Colombia. Únicamente la universidad 1 presenta un 50\% de sus docentes con formación doctoral en esta área del conocimiento. Adicionalmente, el modelo de la universidad 1 presenta un adecuado balance, entre docentes altamente capacitados y formados en las mejores instituciones a nivel internacional, $\mathrm{y}$ una apropiada investigación soportada por laboratorios robustos y suficientes fondos de investigación. Lamentablemente existe un contraste significativo entre el modelo de esta Universidad con el grueso de Facultades de Ingeniería Civil en Colombia.

Las Universidades y los programas académicos en Colombia, en general, deben avanzar en el proceso de autoevaluación, mejoramiento continuo y autorregulación de sus programas de pregrado y posgrado. Se debe fomentar y apoyar la formación docente de calidad, ya sea nacional o internacional, y se debe ser consistente en los presupuestos de investigación y asignaciones salariales en proporción con la calidad y nivel de formación los docentes.

Debido a la importancia del área estructural en el programa de Ingeniería Civil en Colombia, es necesario motivar la formación de docentes en programas de posgrado de calidad nacional o internacional en estructuras. Al mismo tiempo, se requiere que se establezcan políticas nacionales que incentiven y garanticen la vinculación de estos docentes a los programas de Ingeniería Civil de todas las regiones del país, y de esta forma, se pueda descentralizar la oferta académica de calidad en un futuro cercano. Además, se debe propender por garantizar un trato igualitario y equitativo a los egresados de programas doctorales de calidad nacionales e internacionales; y que de esta forma, se contribuya a desacelerar (1) la creciente formación de docentes del área estructural en otras áreas del conocimiento y (2) el marcado acceso de docentes a opciones de formación de posgrados online o semi-precencial de calidad y rigurosidad académica discutibles.

Para finalizar, el panorama general y la importancia del área estructural en programas de Ingeniería Civil en Colombia fueron presentados. Se identificó que existe una elevada demanda de docentes con formación de calidad en estructuras. Por ejemplo, en la universidad 3, únicamente el $44 \%$ de sus docentes tienen formación específica en el área estructural y en términos generales, el 78\% de los docentes adscritos al área estructural, cuentan con un grado de formación máximo como especialistas. Adicionalmente, la combinación de demanda centralizada, baja oferta de programas de calidad nacionales en el área específica del conocimiento, costos elevados de las ofertas académicas de calidad nacionales e internacionales, principalmente, han contribuido a que se presenten las dos tendencias ya reportadas y analizadas; resulta obvio que ambas tendencias son ineficaces en impulsar el desarrollo económico y social de Colombia a través de las actividades de ciencia tecnología e investigación que Colombia requiere, y más aun teniendo presente la elevada amenaza y vulnerabilidad sísmica de la infraestructura colombiana. Por otra parte, es bien sabido que el conocimiento cambia continuamente, y por tanto se requiere una adecuada profundización en cada área del conocimiento en Ingeniería Civil, con la finalidad de eliminar los conocimientos obsoletos y en desuso que, actualmente, se continúan presentando en algunos programas académicos de Ingeniería Civil en el área estructural. Basado en la memoria histórica de Colombia, es necesario tener presente que el camino corto no es, ni será una solución a ninguna problemática, incluyendo la formación profesional. Todo lo anterior, ayuda a explicar el déficit de profesionales con formación doctoral de calidad en el área estructural y que también puede ser aplicable a otras áreas del conocimiento.

\section{Referencias}

[1] Holzer, T.L. and Savage, J.C., Global earthquake fatalities and population, Earthquake Spectra, 29, pp. 155-175, 2013.

[2] AIS 2009, Estudio general de amenaza sísmica de Colombia 2009, Asociación Colombiana de Ingeniería Sísmica, Bogotá, AIS, 2009. 
[3] Garcia, L.E. and Sarria, A., Popayan, Colombia earthquake of March 31, 1983, Engineering Aspects (Preliminary Report), Earthquake Engineering Research Institute (EERI), Palo Alto, Ca. 1983.

[4] Garcia, L.E., The January 25, 1999, Earthquake in the coffee growing region of Colombia: Accelerographic records, structural response and damage, and code compliance and enforcement, 12 WCEE 2000: 12th World Conference on Earthquake Engineering, Auckland, New Zeland, 2809, 2000.

[5] Sánchez-Silva, M., Yamin, L.E. and Caicedo, B., Lessons of the 25 January 1999 earthquake in central Colombia, Earthquake Spectra, 16, pp. 493-510, 2000.

[6] Salinas, D.R., Nonlinear truss analysis of non-ductile rein-forced concrete frames with unreinforced masonry infills, Dissertation/Thesis, Virginia Polytechnic Institute and State University Blacksburg, Virginia, USA, 2016

[7] Pujol, S., Ramirez, J.A. and Sarria, A., Behavior of low-rise reinforced concrete buildings, Concrete International, 22, pp. 40-44, 2000.

[8] Prieto, G., Beroza, G., Barrett, S., López, G. and Florez, M., Earthquake nests as natural laboratories for the study of intermediate-depth earthquake mechanics, Tectonophysics, 570, pp. 42-56, 2012. DOI: 10.1016/j.tecto.2012.07.019.

[9] Taboada, A., Rivera, L.A., Fuenzalida, A., Cisternas, A., Philip, H., Bijwaard, H., Olaya, J. and Rivera, C., Geodynamics of the northern Andes: Subductions and intracontinental deformation (Colombia), Tectonics, 19, pp. 787-813, 2000.

[10] DANE, Censo general 2005-2006, Departamento Nacional de Estadística DANE, 2006.

[11] NSR 2010, El reglamento colombiano de construcción sismo resistente (NSR-10), Asociación Colombiana de Ingeniería Sísmica, Bogotá, AIS, 2010.

[12] AIS 100, Requisitos sísmicos para edificios-Norma AIS 100-81, Asociación Colombiana de Ingeniería Sísmica, Bogotá, AIS, 1981.

[13] SEAOC, Basic design criteria of the recommended lateral force requirements and commentary, Journal of the Structural Division, 98, pp. 1913-1922, 1974.

[14] ATC 3-06, Review and refinement of ATC 3-06 tentative seismic provisions, Report of Technical Committee 9: Regulatory Use, 1980.

[15] Garcia, L.E., Development of the colombian seismic code, Proceedings of the Eight World Conference on Earthquake Engineering, Earthquake Engineering Research Institute (EERI), San Francisco, Ca., USA, 1984.

[16] DEPAE. \& JICA, Estudio para la prevención de desastres en el área metropolitana de Bogotá, en la República de Colombia, 2002.

[17] Garcia, L.E. Seismology and earthquake engineering in Colombia. En: Estrategia Internacional para la Reducción de Desastres EIRD.org doc4558.

[18] Red Sismológica Nacional de Colombia. (RNAC) Disponible en: http://seisan.sgc.gov.co/RSNC

[19] Petroski, H., To forgive design: Understanding failure, Harvard University Press. ISBN-13:978-0674065840, 2014.

[20] Ranking de las mejores universidades de Colombia en 2017. [en línea]. [consultado en: 25 de mayo del 2017]. Disponible en: www.dinero.com.

[21] Soto-Arango, D.E., Los doctorados en Colombia. Un camino hacia la transformación universitaria, Revista Historia de la Educación Latinoamericana, 12(9), pp. 152-195, 2009.

[22] www.dinero.com ¿Cuántos doctores gradúa Colombia en comparación con el resto del mundo?. Educación [en línea]. [consultado en: marzo 15 de 2017] Disponible en: http://www.dinero.com/economia/ articulo/graduados-de-doctorado-en-colombia-y-el-resto-delmundo/242911

[23] Departamento Nacional de Planeación de Colombia, Plan Nacional de Desarrollo 2014-2018, Todos por un nuevo país, ISBN 978-958-834088-3, 2015.

[24] El Espectador.com., El precio de estudiar un doctorado en Colombia Educación. [en línea]. [Consultado en: 25 Feb 2017]. Disponible en: https://www.elespectador.com/noticias/educacion/el-precio-de-estudiarun-doctorado-en-colombia-articulo-681786

[25] Congreso de Colombia, Ley 30 de diciembre 28 de 1992.
[26] Ministerio de Educación Nacional de Colombia, Decreto 1075 del 26 de mayo de 2015.

D.R. Salinas-Guayacundo, es Ing. Civil de la Universidad Pedagógica y Tecnológica de Colombia (UPTC) en Tunja, Colombia en 2003, MSc. en Ing. Civil, Universidad de los Andes, Bogotá, Colombia en 2006 y Dr. of Philosophy (PhD) en Ingeniería Civil (Estructuras) en 2016, del Virginia Polytechnic Institute and State University Blacksburg, Virginia, USA. De 2006 a 2011 orientó cursos de pregrado y posgrado en Universidades de Colombia; y ejerció actividades de consultoría en proyectos de reforzamiento sísmico en Colombia. De agosto del 2007 a junio del 2008 fue invitado por la Universidad HampdenSydney College, en Virginia, USA. Entre los años 2011 y 2016 fue asistente graduado de investigación (GRA), dentro del Structural Engineering and Materials, del Virginia Tech. Desde agosto del 2017 se desempeña como docente catedrático del programa Ingeniería Civil de la Escuela Colombiana de Ingeniería Julio Garavito, Bogotá, Colombia. Adicionalmente, es profesor de posgrados en la Universidad Santo Tomas, Tunja, Colombia. Sus intereses investigativos incluyen: análisis no lineal de pórticos de concreto reforzado, con o sin la presencia de muros de mampostería, sistemas estructurales en acero, concreto y mampostería estructural, ingeniería sísmica, dinámica estructural, diseño basado desempeño, análisis estructural lineal o no lineal usando elementos finitos.

ORCID: 0000-0002-1257-5747 